\title{
Mucinous Carcinoma of the Breast Accompanied by Coarse Calcification
}

\author{
Hitomi Tani ${ }^{1 *}$, Ryusuke Murakami ${ }^{1}$, Tamiko Yoshida ${ }^{1}$, Shinichiro Kumita ${ }^{1}$, \\ Keiko Yanagihara ${ }^{2}$, Shinya Iida ${ }^{2}$, Shinichi Tsuchiya ${ }^{3}$ \\ ${ }^{1}$ Department of Radiology, Graduate School of Medicine, Nippon Medical School, Tokyo, Japan \\ ${ }^{2}$ Department Surgery for Organ Function and Biological Regulation, Graduate School of Medicine, \\ Nippon Medical School, Tokyo, Japan \\ ${ }^{3}$ Division of Diagnostic Pathology, Nippon Medical School Hospital, Tokyo, Japan \\ Email: "h-tsuji@nms.ac.jp
}

Received October 6, 2012; revised November 10, 2012; accepted November 23, 2012

\begin{abstract}
Mucinous carcinoma of the breast has histologically distinct features and constitutes $1 \%-3 \%$ of all breast cancers. It produces variable amounts of extracellular epithelial mucin and clinically its prognosis is far better than for other types of invasive carcinoma. On the other hand, coarse calcification is a common mammographic feature of benign tumors. There have been few reported cases of breast cancer containing coarse calcification. We present a unique 40-year-old female case of mucinous carcinoma of the breast accompanied by coarse calcification on mammography, which was mimicking fibroadenoma and an unusual variant of breast cancer. This case illustrates the importance of bearing in mind that mucinous carcinoma may demonstrate coarse calcification.
\end{abstract}

Keywords: Breast Cancer; Calcification; Mucinous Carcinoma; Mammography

\section{Introduction}

Coarse calcification is a common mammographic feature of benign tumors such as fibroadenoma. Mammographic calcifications associated with malignancy are usually found within ducts and lobules, and do not grow into coarse calcifications. To our knowledge, there have been few reported cases of breast cancer containing coarse calcification. We present a case of mucinous carcinoma of the breast accompanied by coarse calcification that was evident on mammography, and discuss the mammographic and pathological findings.

\section{Case Report}

A 40-year-old woman presented with a mass in the left breast. She had been aware of the mass for about one month. Clinical examination revealed a firm mass $5.0 \mathrm{~cm}$ in diameter in the upper left breast, and some nipple retraction was also evident. Her laboratory data were unremarkable. Mammography demonstrated coarse calcification in the superior lateral quadrant of the left breast, together with diffuse asymmetric density and circumscribed but indistinct calcification (Figure 1). Sonography revealed an ill-defined heteroechoic mass measuring $47 \times 41 \times 17 \mathrm{~mm}$ containing a strongly echoic region 4

"Corresponding author. $\mathrm{mm}$ in diameter. Fine-needle aspiration yielded informative samples containing foamy cells and a few epithelial cells. No malignant cells were evident. Because the tumor was judged to be normal or benign, and histologically similar to hyalinization of a fibroadenoma, tumorectomy was performed.

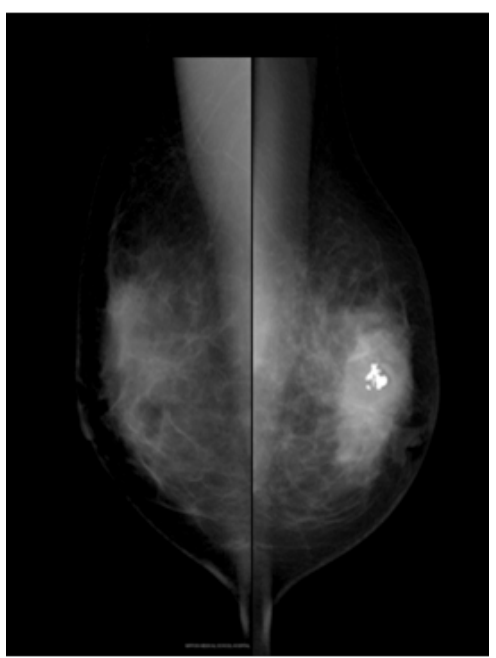

Figure 1. Mammography revealed benign-appearing coarse calcification in the superior lateral quadrant of the left breast, together with asymmetric density. The right breast is normal. 
Macroscopically, the resected specimen contained a circumscribed tumor $75 \times 70 \times 30 \mathrm{~mm}$ in size with a creamy-white cut surface. The tumor extended to the edge of the specimen. Calcification was recognized at the tumor center (Figure 2). Histopathology revealed a mixedtype mucinous carcinoma including scirrhous carcinoma. Immunohistochemical staining revealed positivity for estrogen receptors, negativity for progesterone receptors, and negativity for HER-2. The coarse calcification was present at the center of the tumor and was completely surrounded by mucin pool (Figure 3). After several weeks, the patient underwent modified radical mastectomy with axillary lymph node dissection in view of the positive tumor margin observed after the first operation.

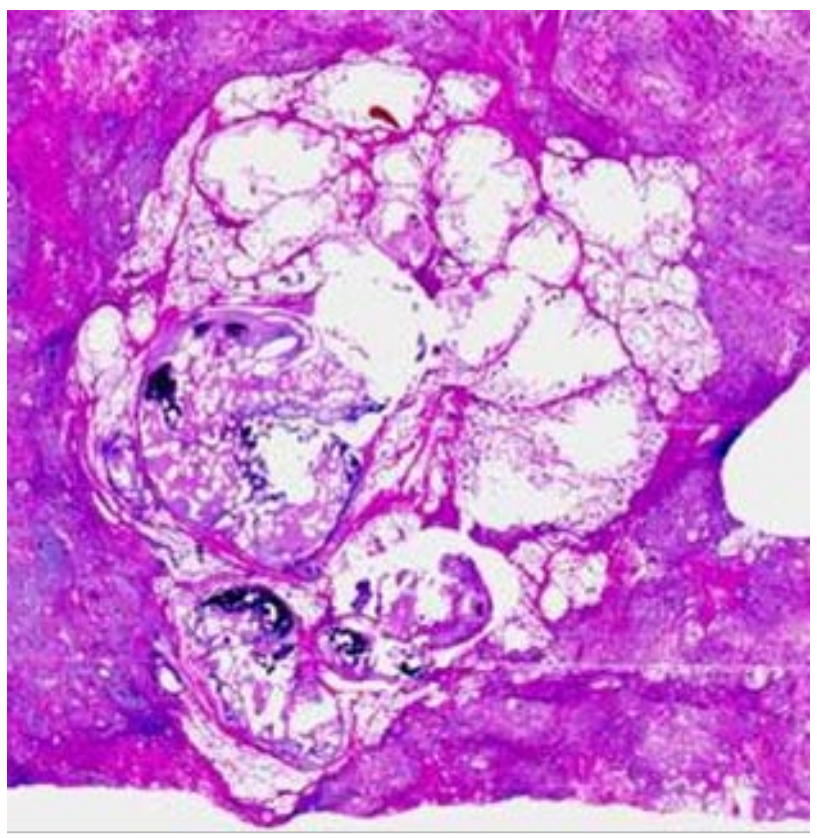

Figure 2. Calcification placed at the tumor center.

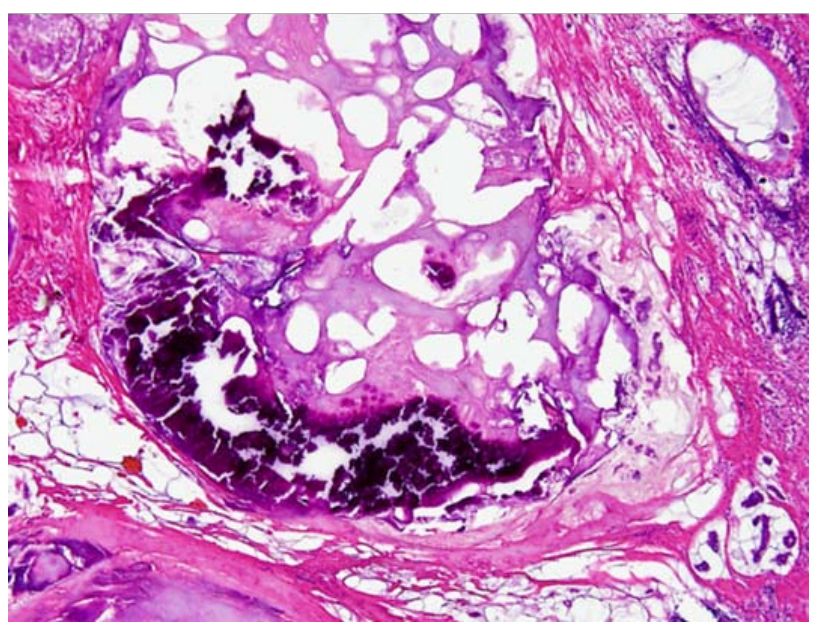

Figure 3. The coarse calcification was seen within the tumor cell clusters and background mucin pool.

\section{Discussion}

Coarse calcification is a well-known feature of fibroadenoma with hyalinization, and is rarely seen in breast cancer. Generally, as the forms of calcification in breast cancer correspond to the structure of the mammary gland, the calcifications will not enlarge theoretically and are generally $<0.5 \mathrm{~mm}$ in size, except for linear and granular calcifications, which need to be identified as benign or malignant. To our knowledge, few reports have described the presence of coarse calcification, not only in mucinous carcinoma of the breast, but any type of breast cancer [1].

Mucinous carcinoma of the breast has histologically distinct features and constitutes $1 \%$ - 3\% of all breast cancers [2]. It produces variable amounts of extracellular epithelial mucin and clinically its prognosis is far better than for other types of invasive carcinoma because it shows less frequent nodal involvement and contains a smaller number of malignant cells [3]. Mucinous carcinoma has two histological subtypes: the pure type in which $>90 \%$ of the tumor has the typical appearance of extracellular mucus-producing areas and the mixed type with $>50 \%$ but $<90 \%$ of a mucinous invasive component $[2,4]$. The present case was mixed-type mucinous carcinoma histologically.

The typical mammographic appearance of mucinous carcinoma is a circular, oval or lobulated mass with a well defined margin. Calcification is sometimes present relatively outside the tumor shadow, together with a circumscribed but indistinct pattern in the case of stromal infiltration or intraductal progression [2].

Some carcinomas, such as thyroid, stomach, and ovary, occasionally contain coarse calcification [5-7]. Although several theories have been proposed, the process of this calcification accompanied by carcinoma has not been identified exactly. In our case, it has been presumed that the decrease in cellular respiration and carbon dioxide production due to insufficient blood supply secondary to ischemia in the mucin pool results in relative alkalinity. Since calcium salts are hardly soluble in alkaline solutions, subsequent precipitation of calcium salts [6]. It has also been suggested that mucin, a glycoprotein, has a predilection for carcification that may act as a nidus.

We report this case to highlight the existence of this unusual variant of mucinous carcinoma of breast. When screening for breast cancer using mammography, careful attention should be paid to coarse calcification associated with masses. It is important to bear in mind that breast cancer, including mucinous carcinoma, may produce coarse calcification.

\section{REFERENCES}

[1] A. Yoshida, H. Tsunoda-Shimizu, M. Kikuchi, Y. Onoda, T. Hamaoka, H. Yagata, et al., "A Case of Mucinous 
Carcinoma with Coarse Calcifications,” Japanese Journal of Breast Cancer, Vol. 23, No. 5, 2008, pp. 429-433.

[2] M. Matsuda, M. Yoshinoto, T. Iwase, K. Takahashi, F. Kasumi, F. Akiyama, et al., "Mammographic and Clinicopathological Features of Mucinous Carcinoma of the Breast,” Breast Cancer, Vol. 7, No. 1, 2000, pp. 65-70. doi:10.1007/BF02967190

[3] A. Memis, N. Ozdemir, M. Parildar, E. E. Ustun and Y. Erhan, "Mucinous (Colloid) Breast Cancer: Mammographic and US Features with Histologic Correlation," European Journal of Radiology, Vol. 35, No. 1, 2000, pp. 39-43. doi:10.1016/S0720-048X(99)00124-2

[4] P. Rao and B. Lyons, "Pure Mucinous Carcinoma of the Breast with Extensive Psammomatous Calcification," Histopathology, Vol. 52, No. 5, 2008, pp. 650-652. doi:10.1111/j.1365-2559.2008.02972.x

[5] C. W. Wu, G. Dionigi, K. W. Lee, P. J. Hsiao, M. C. Paul Shin, K. B. Tsai, et al., "Calcification in Thyroid Nodules Identified on Preoperative Computed Tomography: Patterns and Clinical Significance,” Surgery, Vol. 151, No. 3, 2012, pp. 464-470. doi:10.1016/j.surg.2011.07.032

[6] N. Yildirim Erdogan, O. N. Hüten, F. Bahadir and E. Sander, "Diffuse and Psammomatous Calcification in Intestinal Type Gastric Carcinoma: Report of Two Cases with Literature Review," The Turkish Journal of Gastroenterology, Vol. 22, No. 4, 2011, pp. 414-418.

[7] D. L. Brown, F. C. Laing and W. R. Welch, "Large Calcification in Ovaries Otherwise Normal on Ultrasound," Ultrasound in Obstetrics \& Gynecology, Vol. 29, No. 4, 2007, pp. 438-442. doi:10.1002/uog.3941 